\title{
Correction to: prediction of thrombosis risk in patients with paroxysmal nocturnal hemoglobinuria
}

\author{
Yuzhou Huang ${ }^{1} \cdot$ Xinjian Liu ${ }^{2} \cdot$ Fangfei Chen ${ }^{1} \cdot$ Wenzhe Zhou ${ }^{1} \cdot$ Hongmin $\mathrm{Li}^{1} \cdot$ Zhangbiao Long $^{1} \cdot$ Chen Yang $^{1}$. \\ Miao Chen ${ }^{1}$. Bing Han ${ }^{1}$
}

Published online: 15 November 2019

(C) Springer-Verlag GmbH Germany, part of Springer Nature 2019

\section{Correction to: Annals of Hematology.}

https://doi.org/10.1007/s00277-019-03770-3

The authors determined an error in the affiliation section; it was captured as Department of Hematology, Peking Union Hospital, CAMS \& PUMC, Beijing 100,730, China. The correct affiliation should be Department of Hematology, Peking Union Medical College Hospital, Chinese Academy of Medical Science and Peking Union Medical College, Beijing, 100730, China.

Publisher's note Springer Nature remains neutral with regard to jurisdictional claims in published maps and institutional affiliations.

The online version of the original article can be found at https://doi.org/ 10.1007/s00277-019-03770-3

Bing Han

hanbing_li@sina.com.cn

1 Department of Hematology, Peking Union Medical College Hospital, Chinese Academy of Medical Science and Peking Union Medical College, Beijing 100730, China

2 Department of Hematology, Henan Cancer Hospital, Institute of Hematology of Henan Province, Affiliated Cancer Hospital of Zhengzhou University, 127 Dongming Road, Zhengzhou, China 